\section{JTI}

JOURNAL OF

TRAUMA AND INJURY

\title{
Pulmonary Contusion Similar to COVID-19 Pneumonia
}

\author{
Seung Hwan Lee, M.D. ${ }^{1}$, Sung Youl Hyun, M.D., Ph.D. ${ }^{1,2}$, \\ Yang Bin Jeon, M.D., Ph.D. ${ }^{1,2}$, Jung Nam Lee, M.D. ${ }^{1,2}$, Gil Jae Lee, M.D., Ph.D., ${ }^{1,2}$ \\ ${ }^{1}$ Department of Trauma Surgery, Gachon University Gil Medical Center, Incheon, Korea \\ ${ }^{2}$ Department of Traumatology, Gachon University College of Medicine, Incheon, Korea
}

Received: June 4, 2020

Revised: June 30, 2020

Accepted: June 30, 2020

\section{Correspondence to}

Gil Jae Lee, M.D., Ph.D.

Department of Traumatology, Gachon University College of Medicine, $21 \mathrm{Nam}$ dong-daero 774beon-gil, Namdong-gu, Incheon 21565, Korea

Tel: $+82-32-460-3010$

Fax: $+82-32-460-2372$

E-mail: nonajugi@gilhospital.com
The Coronavirus disease 2019 (COVID-19) has rapidly spread across the world and caused a pandemic. It can be transmitted by an infected person or an asymptomatic carrier and is a highly contagious disease. Prevention and early identification of COVID-19 are important to minimize the transmission of COVID-19. Chest computed tomography (CT) has a high sensitivity for detecting COVID-19, but relatively low specificity. Therefore, chest CT may be difficult to distinguish COVID-19 findings from those of other infectious (notably viral types of pneumonia) or noninfectious disease. Pulmonary contusion has also a lot of similarities on chest CT with COVID-19 pneumonia. We present trauma patients with pulmonary contusion whose CT scans showed findings similar to those of COVID-19, and we report our experience in the management of trauma patients during the COVID-19 pandemic.

Keywords: COVID-19; Pulmonary contusion; Computed tomography; Pneumonia

\section{INTRODUCTION}

Coronavirus disease 2019 (COVID-19) is an infectious spreading disease caused by severe acute respiratory syndrome coronavirus 2 . It was first identified as the cause of a cluster of pneumonia in December 2019 in Wuhan, a city in the Hubei Province of China, and is rapidly spreading around the world, resulted in pandemic declaration by World Health Organization [1-3]. COVID-19 can be transmitted by an infected person or an asymptomatic carrier and is a highly contagious disease [1,2]. Most infected individuals have few or mild symptoms; however, some have serious and critical ill(http://creativecommons.org/licenses/by-nc/4.0/) which permits unrestricted noncommercial use, distribution, and reproduction in any medium, provided the original work is properly cited. 
ness, such as pneumonia or respiratory failure $[4,5]$.

Chest computed tomography (CT) is highly sensitive for diagnosis COVID-19 infection; however, guidelines do not recommend using it for routine screening of COVID-19 [6]. Despite its high sensitivity for diagnosis for COVID-19, chest CT may be difficult to distinguish COVID-19 findings from those of other disease [7]. In particular, pulmonary contusion, which is a bruise of the lung, caused by thoracic trauma, has a lot of similarities on findings of COVID-19 on chest CT, including peripheral subpleural consolidation and ground-glass opacity (GGO) $[8,9]$.

In this paper, we present trauma patients with pulmonary contusion whose CT scans showed findings similar to those of COVID-19, and we report our experience in the management of trauma patients during the COVID-19 pandemic.

\section{CASE REPORT}

A 22-year-old man driver was involved in a rollover motor vehicle accident and transported to the emergency department (ED) via emergency medical services. It was not clear if he was restrained. He was found in rear seat and unconscious at the scene. Immediately upon arrival to the $\mathrm{ED}$, his initial vital signs revealed a blood pressure of $148 / 75 \mathrm{mmHg}$, a pulse of $135 \mathrm{BPM}$, a temperature of $36.8^{\circ} \mathrm{C}$, and an oxygen saturation of $90 \%$ on room air. His Glasgow Coma Scale score was 7 (E 1, V 2, M 4), and he was intubated and ventilated. An extended focused assessment sonography for trauma examination was only positive for free fluid in Morrison's pouch and demonstrated normal lung sliding bilaterally. Two large-bore peripheral lines were established, and a cardiac monitor was applied.

On examination, there was an open fracture of the left distal femur with a laceration approximately $6 \mathrm{~cm} \times$ $2 \mathrm{~cm}$ severely contaminated with the patella exposed on the outside of the wound. A portable chest X-ray study showed small patchy infiltrates in the right lower lung base but did not show a pneumothorax or pleural effusion (Fig. 1A). His pelvis X-ray study was normal. Initial laboratory exams were unremarkable except for a mild leukocytosis of $14,920 / \mathrm{mm}^{3}$ (segment neutrophil of $47.9 \%$ and lymphocyte of 46.6\%), aspartate aminotransferase of $235 \mathrm{U} / \mathrm{L}$, and alanine aminotransferase of $215 \mathrm{U} / \mathrm{L}$.
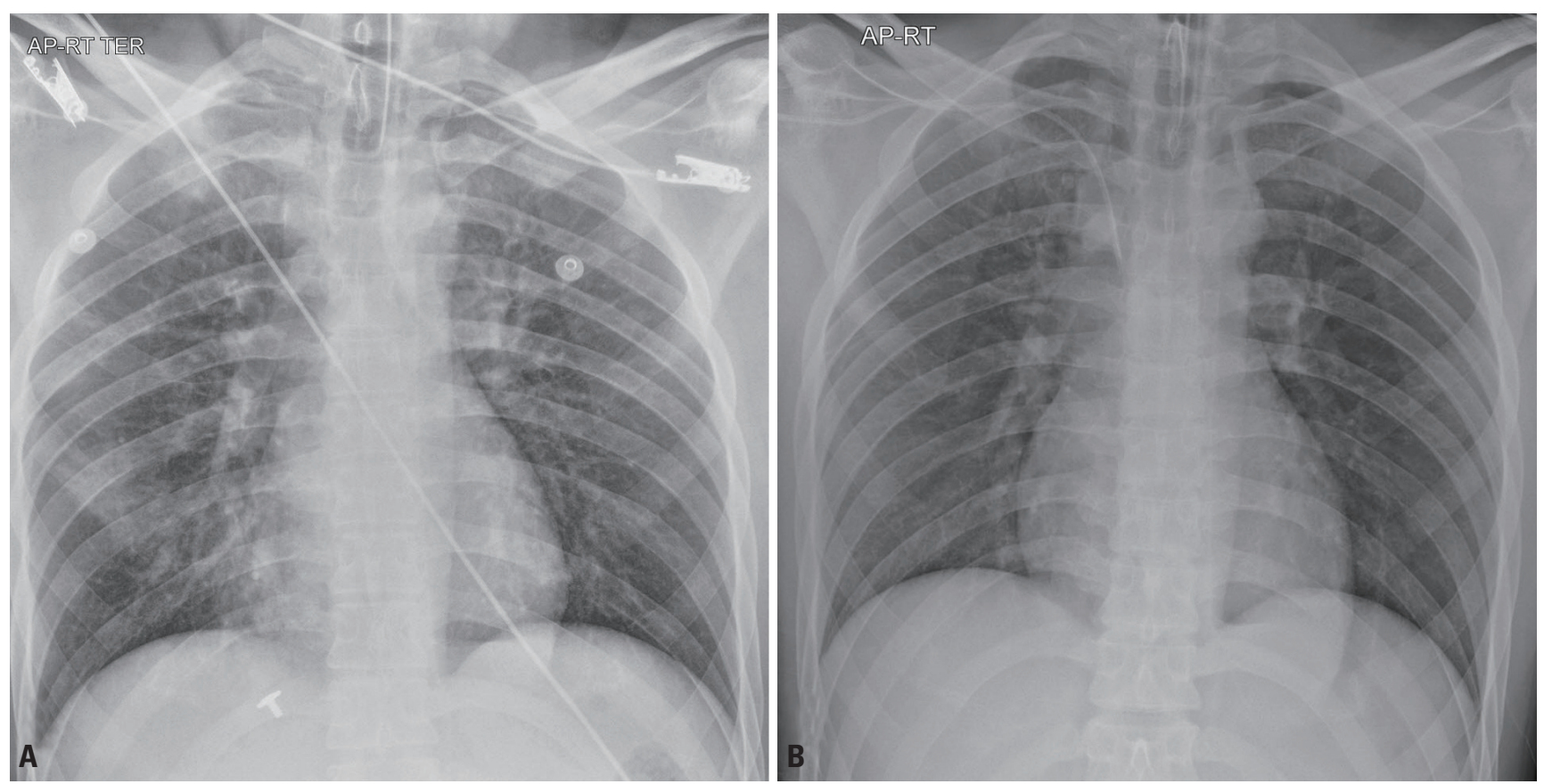

Fig. 1. Chest radiographic images. (A) The initial anteroposterior chest radiograph shows small patchy infiltrates in the right lower lung base. (B) Three days follow-up chest radiograph shows no active lung lesions. 
Brain CT showed hemorrhagic contusions in the left frontal area and fractures in the occipital area. Chest CT scan revealed multifocal GGOs and consolidation with multiple rib fractures in both lungs and small amount pneumothorax in the right lung (Fig. 2). Abdomen and pelvis CT scan showed a grade III liver laceration without active bleeding in segment VIII. X-ray of the left knee revealed comminuted fracture with intraarticular extension of the distal femur.

Despite the CT findings were more suggestive of pulmonary contusion, COVID-19 pneumonia could not be completely ruled out. The patient was transferred in isolation room because epidemiological/clinical information regarding to COVID-19 could not be obtained from the patient and the trauma patient had also potentially highrisk aerosol generating procedures. The real-time reverse transcription polymerase chain reaction (RT-PCR) from nasopharyngeal and oropharyngeal swabs and sputum

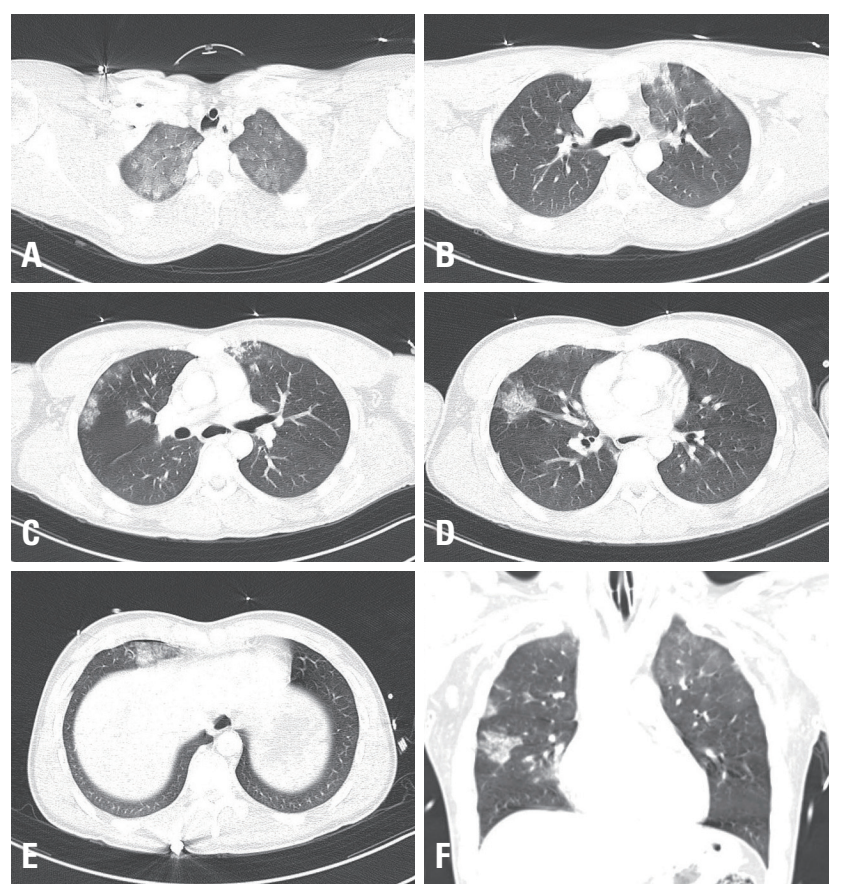

Fig. 2. Chest computed tomographic images. (A) Axial chest computed tomography $(\mathrm{CT})$ image shows bilateral diffuse ground-glass opacities and reticulation. (B-D) Axial chest CT images show multifocal ground-glass opacities and consolidation in both lungs. (E) Axial chest $\mathrm{CT}$ image shows a small amount pneumothorax in the right lung. (F) Coronal chest $\mathrm{CT}$ image shows multiple ground-glass opacities and consolidation in both lungs. was tested, and it revealed all negative. Subsequently, the patient was released from the negative pressure isolation intensive care unit (ICU) and treated as a non-infected trauma patient. The findings on his chest X-ray gradually improved from the third day of hospitalization (Fig. 1B). He underwent orthopedic surgery for left femur fractures 13 days later and was discharged from the hospital after 15 days without complications. The patient had negative RT-PCR tests before discharge.

\section{DISCUSSION}

The COVID-19 has rapidly spread across the world and caused a pandemic, and this situation still continues. While infectious diseases are generally unable to transmit during the incubation period, COVID-19 can show pre-symptomatic transmission [4]. Due to its high transmissibility, prevention and early identification of COVID-19 are important to minimize the transmission of COVID-19.

The standard method of diagnosis is RT-PCR from nasopharyngeal swab and/or oropharyngeal swab $[10,11]$. However, RT-PCR is time-consuming. Chest CT is fast and highly sensitive to diagnosis in individual with high suspicion of COVID-19 infection [7]; however, guidelines do not recommend using it for routine screening of COVID-19 [6]. Despite its high sensitivity in diagnosing COVID-19, due to its low specificity [12], chest CT may be difficult to distinguish COVID-19 findings from those of other disease or pulmonary contusion.

The typical chest CT findings of COVID-19 pneumonia are bilateral GGOs and consolidation with predominant distribution in posterior and peripheral part of the lungs $[13,14]$. However, a recent study reported that $56 \%$ of early patients with COVID-19 had normal chest CT [15]. Pulmonary contusion is a focal parenchymal injury caused by disruption of the capillaries of the alveolar walls and septa, and leakage of blood into the alveolar spaces and interstitium $[9,16]$. Pulmonary contusion can appear as geographic, non-segmental areas of GGOs, nodular opacities, or consolidation on chest CT, and it is not restricted to lobar boundaries $[9,17]$. CT scanning can detect the contusion almost immediately after the injury [18]. 
Unfortunately, the location of GGO and consolidation cannot be used to conclusively differentiate COVID-19 from pulmonary contusion. Nevertheless, the following radiological findings may be helpful in differentiating between COVID-19 and pulmonary contusion. Pleural thickening is detected in 32\% of COVID-19 pneumonia [19], whereas pneumothorax is observed in half of patients with pulmonary contusion and is highly suggestive of pulmonary contusion [20]. Recently, however, there has been a case report of COVID-19 pneumonia accompanied by pneumothorax [21]. Pleural effusion is uncommon in COVID-19; however, large pleural effusion or hemothorax are highly suggestive of pulmonary contusion. Rib and sternal fracture are also commonly found in pulmonary contusion $[15,19,20]$.

Furthermore, clinical course will be useful in differentiating COVID-19 and pulmonary contusion. Most pulmonary contusions occur after diffuse and high energy blunt trauma. Uncomplicated contusions begin clearing within 24 to 48 hours and may resolve after 3-14 days [9,22,23]. In contrast, the lesions of COVID-19 progress rapidly and show concurrently increased number, extent and density of lesions in follow up CT scan [24-27].

Above all, the epidemiological and clinical information can be important clues to differentiate COVID-19 from pulmonary contusion during the initial assessment and management. All trauma patients should be routinely investigated about their exposure history and clinical symptoms related to COVID-19 during pandemic $[28,29]$.

In the present case, we were unable to obtain the epidemiological and clinical information regarding to COVID-19 from the patient due to his altered mental status. Despite CT findings are more suggestive of pulmonary contusion, COVID-19 pneumonia could not be completely ruled out. Because the trauma patient has potentially high-risk aerosol generating procedures, the patient was transferred in isolation room for trauma resuscitation and continued management in the negative pressure isolation ICU until the final negative result was obtained. Attending trauma team wore personal protective equipment (PPE) including N95 respirators, goggles (or face shields), disposable surgical caps, gloves, and disposable fluid-resistant gowns during initial assessment and management according to our guidelines. When carrying out aerosol generating procedures, such as endotracheal intubation and nasopharyngeal/oropharyngeal swabs, our trauma team wore level D PPE. After primary survey, open wound with fracture of the left distal femur was irrigated with normal saline. Cultures were then obtained. The knee joint was manually reduced to its normal position, and then temporary splint was applied to the left extremity. The RT-PCR test finally revealed negative result. Subsequently, the patient was released from isolation and treated as a non-infected trauma patient.

COVID-19 pneumonia and pulmonary contusion each have characteristic features in chest CT images; however, the typical lesions are inconclusive for differentiation of COVID-19 pneumonia and pulmonary contusion. Patients with radiological findings suspicious for COVID-19 should consider prompt RT-PCR testing even in low clinical suspicion patients. All major trauma patients arriving the emergency department should be assumed that they potentially pose a risk of COVID-19 transmission during pandemic, and immediate and comprehensive approach should be considered to minimize risk for COVID-19 transmission.

\section{REFERENCES}

1. Phelan AL, Katz R, Gostin LO. The novel coronavirus originating in Wuhan, China: challenges for global health governance. JAMA 2020 Jan 30. doi: 10.1001/jama.2020.1097 [Epub ahead of print].

2. Li Q, Guan X, Wu P, Wang X, Zhou L, Tong Y, et al. Early transmission dynamics in Wuhan, China, of novel coronavirus-infected pneumonia. N Engl J Med 2020;382:1199-207.

3. Mahase E. COVID-19: WHO declares pandemic because of "alarming levels" of spread, severity, and inaction. BMJ 2020;368:m1036.

4. Guan WJ, Ni ZY, Hu Y, Liang WH, Ou CQ, He JX, et al. Clinical characteristics of coronavirus disease 2019 in China. N Engl J Med 2020;382:1708-1720.

5. Bai Y, Yao L, Wei T, Tian F, Jin DY, Chen L, et al. Presumed asymptomatic carrier transmission of COVID-19. JAMA 2020;323:1406-7.

6. American College of Radiology (ACR). ACR recommendations for the use of chest radiography and computed tomography 
(CT) for suspected COVID-19 infection [Internet]. Reston (VA): ACR 2020 [cited 2020 May 24]. Available from: https://www. acr.org/Advocacy-and-Economics/ACR-Position-Statements/ Recommendations-for-Chest-Radiography-and-CT-for-Suspected-COVID19-Infection.

7. Kanne JP, Little BP, Chung JH, Elicker BM, Ketai LH. Essentials for radiologists on COVID-19: an update- radiology scientific expert panel. Radiology 2020 Feb 27. doi: 10.1148/radiol.2020200527 [Epub ahead of print].

8. Rouhezamin MR, Paydar S, Haseli S. COVID-19 or pulmonary contusion? A diagnostic dilemma. Acad Radiol 2020;27:894-5.

9. Oikonomou A, Prassopoulos P. CT imaging of blunt chest trauma. Insights Imaging 2011;2:281-95.

10. Corman VM, Landt O, Kaiser M, Molenkamp R, Meijer A, Chu DK, et al. Detection of 2019 novel coronavirus (2019-nCoV) by real-time RT-PCR. Euro Surveill 2020;25:2000045.

11. Center for Disease Control and Prevention (CDC). Interim guidelines for collecting, handling, and testing clinical specimens for COVID-19 [Internet]. Washington DC: CDC 2020 [cited 2020 May 24]. Available from: https://www.cdc.gov/coronavirus/2019-ncov/lab/guidelines-clinical-specimens.html.

12. Ai T, Yang Z, Hou H, Zhan C, Chen C, Lv W, et al. Correlation of chest CT and RT-PCR testing in coronavirus disease 2019 (COVID-19) in China: a report of 1014 cases. Radiology 2020 Feb 26. doi: 10.1148/radiol.2020200642 [Epub ahead of print].

13. Yoon SH, Lee KH, Kim JY, Lee YK, Ko H, Kim KH, et al. Chest radiographic and CT findings of the 2019 novel coronavirus disease (COVID-19): analysis of nine patients treated in Korea. Korean J Radiol 2020;21:494-500.

14. Hani C, Trieu NH, Saab I, Dangeard S, Bennani S, Chassagnon G, et al. COVID-19 pneumonia: a review of typical CT findings and differential diagnosis. Diagn Interv Imaging 2020;101:2638.

15. Bernheim A, Mei X, Huang M, Yang Y, Fayad ZA, Zhang N, et al. Chest CT findings in coronavirus disease-19 (COVID-19): relationship to duration of infection. Radiology 2020;295:200463.

16. Wicky S, Wintermark M, Schnyder P, Capasso P, Denys A. Imaging of blunt chest trauma. Eur Radiol 2000;10:1524-38.

17. Kerns SR, Gay SB. CT of blunt chest trauma. AJR Am J Roentgenol 1990;154:55-60.

18. Schild HH, Strunk H, Weber W, Stoerkel S, Doll G, Hein K, et al.
Pulmonary contusion: CT vs plain radiograms. J Comput Assist Tomogr 1989;13:417-20.

19. Shi H, Han X, Jiang N, Cao Y, Alwalid O, Gu J, et al. Radiological findings from 81 patients with COVID-19 pneumonia in Wuhan, China: a descriptive study. Lancet Infect Dis 2020;20: 425-34.

20. Rodriguez RM, Friedman B, Langdorf MI, Baumann BM, Nishijima DK, Hendey GW, et al. Pulmonary contusion in the pan-scan era. Injury 2016;47:1031-4.

21. Aydin S, Öz G, Dumanli A, Balci A, Gencer A. A case of spontaneous pneumothorax in COVID-19 pneumonia. J Surg Res 2020;3:96-101.

22. Rendeki S, Molnár TF. Pulmonary contusion. J Thorac Dis 2019;11(Suppl 2):S141-51.

23. Wanek S, Mayberry JC. Blunt thoracic trauma: flail chest, pulmonary contusion, and blast injury. Crit Care Clin 2004;20:7181.

24. Pan F, Ye T, Sun P, Gui S, Liang B, Li L, et al. Time course of lung changes at chest ct during recovery from coronavirus disease 2019 (COVID-19). Radiology 2020;295:715-21.

25. Li M, Lei P, Zeng B, Li Z, Yu P, Fan B, et al. Coronavirus disease (COVID-19): spectrum of CT findings and temporal progression of the disease. Acad Radiol 2020;27:603-8.

26. Chung M, Bernheim A, Mei X, Zhang N, Huang M, Zeng X, et al. CT imaging features of 2019 novel coronavirus (2019-nCoV). Radiology 2020;295:202-7.

27. Lei J, Li J, Li X, Qi X. CT imaging of the 2019 novel coronavirus (2019-nCoV) pneumonia. Radiology 2020;295:18.

28. Gong Y, Cao X, Mei W, Wang J, Shen L, Wang S, et al. Anesthesia considerations and infection precautions for trauma and acute care cases during the COVID-19 pandemic. Anesth Analg 2020 Apr 24. doi: 10.1213/ANE.0000000000004913 [Epub ahead of print].

29. Michetti CP, Burlew CC, Bulger EM, Davis KA, Spain DA; Critical Care and Acute Care Surgery Committees of the American Association for the Surgery of Trauma. Performing tracheostomy during the COVID-19 pandemic: guidance and recommendations from the Critical Care and Acute Care Surgery Committees of the American Association for the Surgery of Trauma. Trauma Surg Acute Care Open 2020;5:e000482. 